\title{
Sleep disordered breathing and fibroblast growth factor 23 in the Hispanic Community Health Study/Study of Latinos
}

\author{
Rupal Mehta ${ }^{\mathrm{a}, \mathrm{b}, \mathrm{c}, *}, \mathrm{Xuan}_{\mathrm{Cai}^{\mathrm{b}}}^{\mathrm{b}}$, Alexander Hodakowski ${ }^{\mathrm{b}}$, Bharat Thyagarajan ${ }^{\mathrm{d}}$, Donglin Zenge, \\ Phyllis C. Zee ${ }^{\mathrm{f}}$, William K. Wohlgemuth ${ }^{\mathrm{g}, \mathrm{k}}$, Susan Redline ${ }^{\mathrm{h}, \mathrm{l}}$, James P. Lash ${ }^{\mathrm{i}}$, Myles Wolf ${ }^{\mathrm{j}}$, \\ Tamara Isakova ${ }^{\mathrm{a}, \mathrm{b}}$ \\ a Division of Nephrology and Hypertension, Department of Medicine, Northwestern University Feinberg School of Medicine, Chicago, IL, USA \\ ${ }^{\mathrm{b}}$ Center for Translational Metabolism and Health, Institute for Public Health and Medicine, Northwestern University Feinberg School of Medicine, Chicago, IL, USA \\ ${ }^{\mathrm{c}}$ Jesse Brown Veterans Administration Medical Center, Chicago, IL, USA \\ ${ }^{\mathrm{d}}$ Department of Laboratory Medicine and Pathology, Division of Molecular Pathology and Genomics, University of Minnesota, Minneapolis, MN, USA \\ e Department of Biostatistics, University of North Carolina Gillings School of Global Public Health, Chapel Hill, NC, USA \\ ${ }^{\mathrm{f}}$ Division of Sleep Medicine, Department of Neurology, Northwestern University Feinberg School of Medicine, Chicago, IL, USA \\ ${ }^{g}$ Division of Neurology, Bruce W. Carter Department of Veterans Affairs Medical Center, Miami, FL, USA \\ ${ }^{\mathbf{h}}$ Division of Sleep and Circadian Disorders, Department of Medicine, Brigham and Women's Hospital, Boston, USA \\ ${ }^{i}$ Division of Nephrology, Department of Medicine, University of Illinois at Chicago, Chicago, IL, USA \\ ${ }^{\mathbf{j}}$ Division of Nephrology, Department of Medicine, Duke University, Durham, NC, USA \\ ${ }^{\mathrm{k}}$ Division of Psychology, Bruce W. Carter Department of Veterans Affairs Medical Center, Miami, FL, USA \\ ${ }^{1}$ Division of Sleep and Circadian Disorders, Department of Neurology, Brigham and Women's Hospital, Boston, USA
}

\section{A R T I C L E I N F O}

Keywords:

Fibroblast growth factor 23

Hypoxia

Sleep disordered breathing

Chronic kidney disease

Osteocyte

\begin{abstract}
A B S T R A C T
Preclinical data suggest that hypoxia stimulates fibroblast growth factor 23 (FGF23) transcription and cleavage in osteocytes, resulting in elevated circulating c-terminal (cFGF23) levels but normal intact FGF23 (iFGF23) levels. We conducted a case-control study within the Hispanic Community Health Study/Study of Latinos to investigate whether sleep disordered breathing, as a model of hypoxemia, is independently associated with elevated cFGF23 levels in the general population and with elevated cFGF23 and iFGF23 levels in patients with chronic kidney disease (CKD), in whom FGF23 cleavage may be impaired. Cases $(n=602)$ had severe sleep disordered breathing defined as an apnea/hypopnea index (AHI) of $\geq 30$. Controls without severe sleep disordered breathing $(\mathrm{n}=602)$ were matched for sex and CKD stage. The median AHI in the cases was 45.8 (IQR 35.5-62.5) compared to 2.6 (IQR 0.6-8.2) in the controls. Cases had higher cFGF23 levels than controls (66.2 RU/mL, IQR 52.8-98.4 vs. 61.2 RU/mL, IQR 49.5-80.1, p value < 0.001). There were no differences in iFGF23 levels between cases and controls. In adjusted linear regression and multinomial regression analyses, body mass index attenuated the relationship between severe sleep disordered breathing and cFGF23 levels. No significant relationships were seen in analyses of severe sleep disordered breathing and iFGF23 levels or in analyses of iFGF23 and cFGF23 stratified by CKD status. Additional studies using other models of intermittent and chronic hypoxia are needed to confirm whether hypoxia stimulates FGF23 transcription in humans and to determine the impact on iFGF23 levels in CKD.
\end{abstract}

\section{Introduction}

Fibroblast growth factor 23 (FGF23) is an osteocyte-derived hormone that regulates systemic phosphate and vitamin D homeostasis [1]. Chronic kidney disease (CKD) represents the most common setting of secondary FGF23 excess. FGF23 levels rise early in CKD and FGF23 levels may be thousands-fold higher than in CKD by the time end stage renal disease occurs [2]. Preclinical data demonstrate direct toxic effects of FGF23 on cardiac myocytes through activation of fibroblast growth factor receptor 4 [3, 4]. Results from observational studies are

\footnotetext{
Abbreviations: FGF23, fibroblast growth factor 23; cFGF23, c-terminal fibroblast growth factor 23; iFGF23, intact fibroblast growth factor 23; CKD, chronic kidney disease; HCHS/SOL,

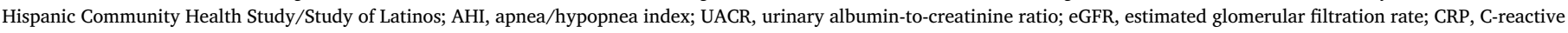
protein; OR, odds ratio

* Corresponding author at: 633 North Saint Clair, 18-081, Chicago, IL 60611, USA.

E-mail address: rupal.mehta@northwestern.edu (R. Mehta).
} 
corroborative: higher FGF23 levels are strongly associated with left ventricular hypertrophy, cardiovascular events and mortality in patients with CKD [5-12]. Collectively, these findings have spurred investigations to identify modifiable factors that regulate circulating FGF23 levels.

Studies in animal models demonstrate that the balance between FGF23 transcription and post-translational cleavage of newly synthesized FGF23 protein determines levels of circulating bioactive FGF23 [13]. FGF23 transcription is upregulated in response to known stimuli and in health, this is matched by an increase in post-translational FGF23 cleavage [13, 14]. Thus, circulating levels of biologically-active hormone, which are measured by intact assays (iFGF23), stay within the normal range, but C-terminal inactive fragments are elevated, which are measured by assays that detect both the biologically-active intact hormone and C-terminal fragments (cFGF23) [13-15]. Iron deficiency and inflammation are two recently discovered stimuli of FGF23 transcription that are accompanied by parallel increases in FGF23 cleavage, resulting in elevations of cFGF23 but not iFGF23 levels. In CKD, FGF23 cleavage may be impaired such that stimuli that upregulate FGF23 transcription in osteocytes produce elevations in both cFGF23 and iFGF23 [1]. Preclinical data demonstrate that hypoxia may be a novel stimulus of FGF23 transcription [16]. However, few clinical studies have investigated the effect of hypoxemia on circulating FGF23 levels in health and in CKD.

Sleep disordered breathing is a common cause of nocturnal hypoxemia that is strongly associated with increased risk of cardiovascular disease in the general population and in patients with CKD [17-25]. We conducted a nested case-control study within a large prospective cohort of individuals with and without CKD to test the hypothesis that individuals with severe sleep disordered breathing would have higher baseline cFGF23 levels compared to individuals without severe sleep disordered breathing. Given that post-translational cleavage of FGF23 may be impaired in CKD [15, 26], in exploratory analyses, we tested the hypothesis that individuals with sleep disordered breathing and CKD, defined as an estimated glomerular filtration rate $<60 \mathrm{~mL} / \mathrm{min}$ / $1.73 \mathrm{~m}^{2}$, would have elevations in both cFGF23 and iFGF23 levels.

\section{Methods}

\subsection{Study population and design}

The Hispanic Community Health Study/Study of Latinos (HCHS/ SOL) is an ongoing multi-center prospective cohort study of risk factors for and the prevalence of cardiovascular, pulmonary and chronic diseases in the Hispanic/Latino population [27, 28]. The HCHS/SOL study population included 16,415 participants aged $18-74$ years who were recruited between 2008 and 2011 from four field centers across the United States: Chicago, IL; Bronx, NY, Miami, FL; San Diego, CA. The sampling design and implementation of the HCHS/SOL were previously described [27, 28]. All participants gave written informed consent, and the study was approved by the Institutional Review Board at each participating field center [27, 28].

Of the total HCHS/SOL study population, 14,440 individuals underwent a sleep study at their baseline visit with sufficient somnography data for analyses [29]. For this case-control study [30], we identified 602 individuals who met the severe sleep disordered breathing case definition and who had plasma available for additional measurements. We then selected 602 controls, frequency-matched for sex and CKD stage, who did not have severe sleep disordered breathing. Our total population included 1204 individuals in whom we measured baseline plasma cFGF23, iFGF23 and phosphate (Fig. 1).

\subsection{Primary exposure}

The primary exposure was the presence of severe sleep disordered breathing. Participants were instructed on use of a Type 3 sleep apnea monitor for overnight recording at their baseline clinic visit, when participants also had their blood drawn [29]. This monitor measures airflow via nasal cannula, pulse rate, snoring levels, and hemoglobin oxygen saturation via transcutaneous oximetry [29]. All sleep records were scored at a central sleep reading center by a certified polysomnologist, who manually edited artifacts, identified periods of sleep, and interpreted the oxyhemoglobin desaturation associated with each respiratory event [29]. Respiratory events were defined as a $50 \%$ or greater reduction in airflow that lasted $\geq 10 \mathrm{~s}$ [29]. Apnea/hypopnea index (AHI) $\geq 30$ was defined as $>30$ respiratory events per estimated sleep hour with $3 \%$ desaturation. Within and between scorer reliability was high for the AHI (intra-class and inter-class correlation coefficients $>0.95$ ) [29]. Severe sleep disordered breathing was defined as $\mathrm{AHI} \geq 30$ on overnight sleep testing.

\subsection{Outcomes}

Our primary outcomes were plasma cFGF23 and iFGF23 levels. We measured FGF23 using Quidel's (formerly known as Immutopics) cFGF23 and iFGF23 assays (San Clemente, California, Quidel) at the HCHS/SOL Central Laboratory at the University of Minnesota after a single thaw of frozen samples. To quantify precision, we measured in a blinded manner a random selection of $10 \%$ of samples in duplicate. The mean intra-assay coefficients of variation for CFGF23 and iFGF23 were $4.4 \%$ and $6.6 \%$. The inter-assay coefficients of variation ranged from 4.8-8.3\% for cFGF23 and 6.1-6.4\% for iFGF23.

\subsection{Covariates}

Information on participant demographics, medication use and clinical data was collected at the baseline visit [27, 28]. Resting blood pressure was measured via standardized protocols. Diabetes was defined as one of the following: fasting plasma glucose level $\geq 126 \mathrm{mg}$ / $\mathrm{dL}$, a 2-hour post-load glucose level $\geq 200 \mathrm{mg} / \mathrm{dL}$, hemoglobin A1C level $\geq 6.5 \%$, or documented use of hypoglycemic agents on their medication inventory [31, 32]. The centralized laboratory measured serum creatinine and calcium, and urinary albumin-to-creatinine ratio (UACR) via standard assays. Phosphate was measured in plasma on a Roche Cobas 6000 Chemistry Analyzer (Roche Diagnostics Corporation) using a colormetric method. For plasma phosphate, the laboratory intra-assay CV was $0.8 \%$ at a concentration of $6.1 \mathrm{mg} / \mathrm{dL}$ and the interassay CV was $1.9 \%$ at a concentration of $3.7 \mathrm{mg} / \mathrm{dL}$. CKD was defined as an estimated glomerular filtration rate (eGFR) $<60 \mathrm{~mL} / \mathrm{min} / 1.73 \mathrm{~m}^{2}$ using the creatinine-based Chronic Kidney Disease Epidemiology Collaboration Cystatin $\mathrm{C}$ equation [33]. The amount of missing data for covariates was minimal and less than $<0.5 \%$.

\subsection{Statistical analysis}

We used standard descriptive statistics to summarize and compare baseline characteristics of our study population according to presence of severe sleep disordered breathing at study enrollment.

To test the association of sleep disordered breathing with FGF23 levels, we fit separate models for cFGF23 and iFGF23. First, we used multinomial logistic regression analyses with cFGF23 and iFGF23 expressed in ascending quartiles, as previously done [7, 34]. We then examined FGF23 as a continuous outcome variable in linear regression models, for which we log transformed cFGF23 and iFGF23 levels because they were not normally distributed. We hierarchically adjusted all models for potential confounding factors, including demographics (age and sex), CKD risk factors (eGFR, albuminuria and plasma phosphate), comorbidities (blood pressure, smoking, diabetes, and coronary heart disease), and inflammation (C-reactive protein [CRP]). In our final model, we further adjusted for body mass index.

Given that FGF23 cleavage may be impaired in CKD [15, 26], we performed pre-planned exploratory subgroup analyses in those with 


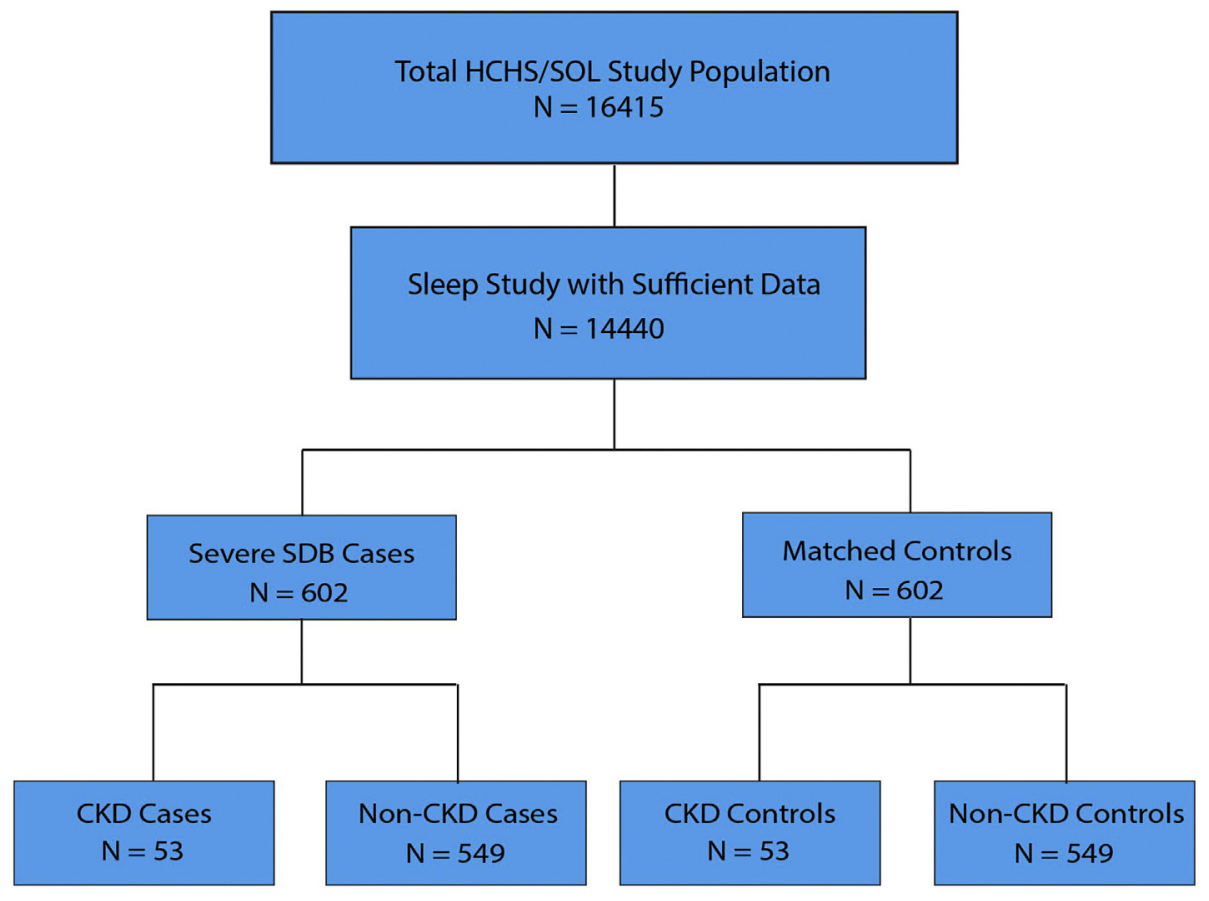

Fig. 1. Case control study design

Controls are frequency matched for sex and CKD stage.

Abbreviations: HCHS/SOL, Hispanic Community Health Study/Study of Latinos; N, number; SDB, sleep disordered breathing; CKD, chronic kidney disease.

and without CKD. In analyses restricted to those with or without CKD, we compared those with severe sleep disordered breathing to controls using standard descriptive statistics. We used linear regression to investigate the associations of severe sleep disordered breathing and cFGF23 and iFGF23 expressed as a continuous variable. We used a similar hierarchical modeling approach to that used in our total population.

In sensitivity analyses, we replaced body mass index with waist circumference, which may be a more accurate measure of adiposity, in our multinomial and linear regression models.

Analyses were performed using SAS version 9.4 (Cary, NC, USA). Two-sided $\mathrm{p}$ values $<0.05$ were considered statistically significant.

\section{Results}

\subsection{Associations of sleep disordered breathing with FGF23 levels}

Table 1 presents clinical and demographic characteristics of the $1204 \mathrm{HCHS} / \mathrm{SOL}$ participants according to presence of severe sleep disordered breathing. The median $\mathrm{AHI}$ in individuals with severe sleep disordered breathing was 45.8 (IQR 35.5-62.5) compared to 2.6 (IQR 0.6-8.2) in the control group. Compared to controls, individuals with severe sleep disordered breathing were older and had higher prevalence of diabetes. Individuals with severe sleep disordered breathing also had higher levels of inflammation and were more obese compared to controls (CRP $3.4 \mathrm{mg} / \mathrm{L}$, IQR $1.8-6.9$ vs. $2.0 \mathrm{mg} / \mathrm{L}$, IQR $0.9-4.8$, p value $<0.001$; body mass index $35.2 \pm 6.6 \mathrm{~kg} / \mathrm{m}^{2}$ vs. $29.9 \pm 5.8 \mathrm{~kg} /$ $\mathrm{m}^{2}$, p value $<0.001$, Table 1 ). Levels of cFGF23 were slightly higher in individuals with severe sleep disordered breathing compared to controls (66.2 RU/mL, IQR 52.8-98.4 vs. $61.2 \mathrm{RU} / \mathrm{mL}$, IQR 49.5-80.1, p value $<0.001$, Table 1 ). iFGF23 levels did not differ between individuals with severe sleep disordered breathing and those without (Table 1).

We investigated the associations of sleep disordered breathing with ascending FGF23 quartiles using multinomial logistic regression analyses. In unadjusted analyses, individuals with severe sleep disordered breathing had higher odds of belonging to cFGF23 Quartile 4 versus
Table 1

Characteristics of total population according to sleep disordered breathing status.

\begin{tabular}{|c|c|c|c|}
\hline & Severe SDB & Severe SDB & $\mathrm{p}$ value \\
\hline & $\begin{array}{l}\text { Yes } \\
\mathrm{N}=602\end{array}$ & $\begin{array}{l}\text { No } \\
\mathrm{N}=602\end{array}$ & \\
\hline Age, years & $52.8 \pm 11.2$ & $48.7 \pm 14.5$ & $<0.001$ \\
\hline Female, \% & $211(35.1)$ & $210(34.9)$ & 1.0 \\
\hline BMI, $\mathrm{kg} / \mathrm{m}^{2}$ & $35.2 \pm 6.6$ & $29.9 \pm 5.8$ & $<0.001$ \\
\hline $\mathrm{SBP}, \mathrm{mm} \mathrm{Hg}$ & $129.5 \pm 18.5$ & $125.8 \pm 18.0$ & $<0.001$ \\
\hline Hypertension, $\mathrm{N}(\%)$ & $300(49.8)$ & $219(36.4)$ & $<0.001$ \\
\hline Diabetes, $N(\%)$ & $232(38.5)$ & $139(23.1)$ & $<0.001$ \\
\hline CHD, N (\%) & $44(7.3)$ & $30(5.0)$ & 0.09 \\
\hline $\mathrm{eGFR}, \mathrm{mL} / \mathrm{min} / 1.73 \mathrm{~m}^{2}$ & $93.7 \pm 22.8$ & $97.2 \pm 25.2$ & 0.01 \\
\hline Albuminuria, N (\%) & $126(20.9)$ & $77(12.8)$ & $<0.001$ \\
\hline Albumin/creatinine ratio, $\mathrm{mg} / \mathrm{g}$ & $8.6(5.2-21.6)$ & $6.7(4.4-13.1)$ & $<0.001$ \\
\hline Phosphate, mg/dL & $3.3 \pm 0.5$ & $3.3 \pm 0.5$ & 0.4 \\
\hline $\mathrm{CRP},(\mathrm{mg} / \mathrm{L})$ & $3.4(1.8-6.9)$ & $2.0(0.9-4.8)$ & $<0.001$ \\
\hline C-terminal FGF23, RU/mL & $66.2(52.8-98.4)$ & $61.2(49.5-80.1)$ & $<0.001$ \\
\hline intact FGF23, pg/mL & $53.2(42.8-67.7)$ & $53.2(42.8-71.2)$ & 0.8 \\
\hline Apnea/hypopnea index & $45.8(35.5-62.5)$ & $2.6(0.6-8.2)$ & $<0.001$ \\
\hline
\end{tabular}

Results are reported as means \pm standard deviation, proportions or medians with interquartile ranges.

Abbreviations: N, number; SDB, sleep disordered breathing; BMI, body mass index; SBP, systolic blood pressure; CHD, cardiovascular heart disease; eGFR, estimated glomerular filtration rate; CRP, C-reactive protein FGF23, fibroblast growth factor 23.

Quartile 1 (cFGF23 Quartile 4 vs. Quartile 1 Odds Ratio [OR] 2.02, CI 1.46-2.80 Table 2). After adjustment for demographics, CKD-associated risk factors, comorbidities, and CRP, individuals with severe sleep disordered breathing continued to have higher odds of belonging to cFGF23 Quartile 4 versus Quartile 1 (cFGF23 Quartile 4 vs. Quartile 1 OR 1.93, CI 1.31-2.86 Table 2). After adjustment for body mass index, the association was attenuated but remained significant (cFGF23 Quartile 4 vs. Quartile 1 OR 1.70, CI 1.12-2.59, Table 2). We did not appreciate a significant relationship between severe sleep disordered 
Table 2

Adjusted associations of severe sleep disordered breathing presence with cFGF23 expressed as quartiles.

\begin{tabular}{|c|c|c|c|c|c|}
\hline \multirow[b]{2}{*}{$\begin{array}{l}\text { AHI } \geq 30 \\
\text { vs. } \\
\text { AHI }<30\end{array}$} & \multirow[b]{2}{*}{ Total N } & \multicolumn{4}{|c|}{ cFGF23 quartiles (RU/mL) } \\
\hline & & $\begin{array}{l}\text { Quartile } 1 \\
<50.5 \\
\mathrm{~N}=302\end{array}$ & $\begin{array}{l}\text { Quartile } 2 \\
50.5-63.15 \\
N=300\end{array}$ & $\begin{array}{l}\text { Quartile } 3 \\
63.16-87.95 \\
N=301\end{array}$ & $\begin{array}{l}\text { Quartile } 4 \\
>87.96 \\
\mathrm{~N}=301\end{array}$ \\
\hline Unadjusted & 1204 & REF & $\begin{array}{l}1.22 \\
(0.89-1.69)\end{array}$ & $1.32(0.95-1.81)$ & $\begin{array}{l}2.02 \\
(1.46-2.80)\end{array}$ \\
\hline Model 1 & 1204 & REF & $\begin{array}{l}1.21 \\
(0.87-1.68)\end{array}$ & $1.29(0.93-1.79)$ & $\begin{array}{l}1.98 \\
(1.41-2.77)\end{array}$ \\
\hline Model 2 & 1203 & REF & $\begin{array}{l}1.27 \\
(0.91-1.77)\end{array}$ & $1.43(1.01-2.01)$ & $\begin{array}{l}2.23 \\
(1.53-3.24)\end{array}$ \\
\hline Model 3 & 1200 & REF & $\begin{array}{l}1.24 \\
(0.89-1.74)\end{array}$ & $1.37(0.96-1.93)$ & $\begin{array}{l}2.10 \\
(1.43-3.08)\end{array}$ \\
\hline Model 4 & 1200 & REF & $\begin{array}{l}1.19 \\
(0.84-1.69)\end{array}$ & $1.41(0.99-2.02)$ & $\begin{array}{l}1.93 \\
(1.31-2.86)\end{array}$ \\
\hline Model 5 & 1197 & REF & $\begin{array}{l}1.08 \\
(0.75-1.57)\end{array}$ & $1.39(0.95-2.03)$ & $\begin{array}{l}1.70 \\
(1.12-2.59)\end{array}$ \\
\hline
\end{tabular}

Model 1 adjust for age and gender.

Model 2 adjusts for covariates in Model 1 plus plasma phosphate, eGFR and UACR.

Model 3 adjusts for covariates in Model 2 plus hypertension, diabetes presence, cardiovascular disease, cigarette use.

Model 4 adjusts for covariates in Model 3 plus C-reactive protein.

Model 5 adjusts for covariates in Model 4 plus body mass index.

Abbreviations: FGF23, fibroblast growth factor 23; N, number; eGFR, estimated glomerular filtration rate; UACR, urinary albumin-to-creatinine ratio.

breathing and iFGF23 Quartiles (iFGF23 Quartile 4 vs. Quartile 1 OR 0.76 , CI 0.52-1.11).

In the unadjusted linear regression analyses, severe sleep disordered breathing was associated with higher cFGF23 levels expressed as a continuous variable ( $\beta$-estimate 0.12 , CI $0.04-0.19$, p value 0.002 , Table 3). The relationship remained significant after adjustment for demographics, CKD risk factors, comorbidities, and CRP ( $\beta$ estimate 0.08 , CI $0.02-0.15, \mathrm{p}$ value 0.02 ). However, the association became non-significant after the addition of body mass index ( $\beta$ estimate 0.07 , CI $-0.005-0.14$, p value 0.07 , Table 3 ). We did not identify a significant relationship between severe sleep disordered breathing and iFGF23 levels (Table 3).

\subsection{Subgroup analyses by CKD status}

Of the 1204 individuals in the total population, 106 met criteria for CKD. Clinical characteristics of individuals by severe sleep disordered breathing status in individuals with and without CKD are presented in Supplemental Tables 1 and 2. The mean eGFR in those with CKD and severe sleep disordered breathing was $46.3 \pm 13.3 \mathrm{~mL} / \mathrm{min} / 1.73 \mathrm{~m}^{2}$ and in those with CKD without severe sleep disordered breathing was $45.0 \pm 13.5 \mathrm{~mL} / \mathrm{min} / 1.73 \mathrm{~m}^{2}$ (Supplemental Table 1). Individuals with CKD and severe sleep disordered breathing had significantly higher body mass index and CRP than those with CKD but without severe sleep disordered breathing (body mass index $35.4 \pm 6.7 \mathrm{~kg} / \mathrm{m}^{2}$ vs. $30.0 \pm 6.8 \mathrm{~kg} / \mathrm{m}^{2}$, p value < 0.001; CRP $6.1 \mathrm{mg} / \mathrm{L} \mathrm{IQR} 2.7-11.6$ vs. $3.0 \mathrm{mg} / \mathrm{L}$ IQR 1.4-5.8, p value 0.001, Supplemental Table 1). cFGF23 and iFGF23 levels were also higher in those with severe sleep disordered breathing than in those without severe sleep disordered breathing, however this did not reach significance (Supplemental Table 1). In multivariable analyses that were restricted to individuals with CKD, severe sleep disordered breathing was not associated with higher cFGF23 levels or iFGF23 levels (Supplemental Table 3).

\subsection{Sensitivity analyses}

Because waist circumference may be a more accurate measurement of adiposity compared to BMI, we replaced body mass index with waist circumference in our multinomial regression and linear regression models,. Our results were qualitatively similar. In our adjusted multinomial regression analyses, the association between severe sleep disordered breathing and cFGF23 quartiles remained significant but was attenuated after adjustment for waist circumference (cFGF23 Quartile 4 vs. Quartile 1 OR 1.62, CI 1.07-2.46). In our linear regression analyses investigating the associations between severe sleep disordered breathing and higher cFGF23 levels, similar to our models with body mass index, the association became non-significant after the addition of waist circumference ( $\beta$ estimate 0.06 , CI $-0.01-0.13$, $p$ value 0.11 ).

\section{Discussion}

In a sample drawn from a large cohort of Hispanic/Latino patients with and without CKD, we demonstrated that individuals with severe sleep disordered breathing had slightly higher levels of cFGF23 than those without severe sleep disordered breathing, but no difference in iFGF23 levels. Body mass index or waist circumference were the main confounders that attenuated the sleep disordered breathing-cFGF23 relationship in both linear and multinomial logistic regression analyses. We did not appreciate any relationship with severe sleep disordered breathing presence and iFGF23 in the total population or in the subgroup with CKD. These results are broadly consistent with our hypotheses and suggest that in addition to iron deficiency and inflammation, sleep disordered breathing may be another clinical entity in which we see upregulation of FGF23 transcription in osteocytes and an elevation in cFGF23 but not iFGF23 levels.

In vivo and in vitro studies demonstrate that hypoxia regulates

Table 3

Adjusted associations of severe sleep disordered breathing with cFGF23 and iFGF23 levels expressed as continuous variables.

\begin{tabular}{|c|c|c|c|c|c|c|c|}
\hline \multirow[b]{2}{*}{$\mathrm{AHI} \geq 30$ vs. $\mathrm{AHI}<30$} & \multirow[b]{2}{*}{ Total N } & \multicolumn{3}{|l|}{ c-terminal FGF23 } & \multicolumn{3}{|l|}{ iFGF23 } \\
\hline & & Parameter Estimate & $95 \%$ CI & p-Value & Parameter estimate & $95 \% \mathrm{CI}$ & p-Value \\
\hline Unadjusted & 1204 & 0.12 & $0.04-0.19$ & 0.002 & -0.02 & $-0.08-0.03$ & 0.4 \\
\hline Model 1 & 1204 & 0.10 & $0.03-0.17$ & 0.006 & -0.03 & $-0.09-0.02$ & 0.3 \\
\hline Model 2 & 1203 & 0.09 & $0.03-0.16$ & 0.006 & -0.03 & $-0.09-0.02$ & 0.2 \\
\hline Model 3 & 1200 & 0.08 & $0.02-0.15$ & 0.01 & -0.03 & $-0.08-0.02$ & 0.2 \\
\hline Model 4 & 1200 & 0.08 & $0.02-0.15$ & 0.02 & -0.03 & $-0.08-0.03$ & 0.4 \\
\hline Model 5 & 1197 & 0.07 & $-0.005-0.14$ & 0.07 & -0.03 & $-0.09-0.03$ & 0.3 \\
\hline
\end{tabular}

Model 1 adjust for age and gender.

Model 2 adjusts for covariates in Model 1 plus plasma phosphate, eGFR and UACR.

Model 3 adjusts for covariates in Model 2 plus hypertension, diabetes presence, cardiovascular disease, cigarette use.

Model 4 adjusts for covariates in Model 3 plus C-reactive protein.

Model 5 adjusts for covariates in Model 4 plus body mass index.

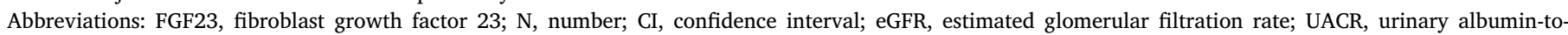
creatinine ratio. 
FGF23 transcription [16]. When UMR-106 cells are grown in an oxygen deprived environment, expression of FGF23 mRNA increases. This parallels increases in HIF-1 $\alpha$ protein expression, making it plausible that the appreciable increase in FGF23 mRNA expression is in response to cell hypoxia [16]. In vivo models support these findings. When animals are exposed to a hypobaric atmosphere, cFGF23 increases by six fold, but iFGF23 remains unchanged [16]. This indicates that hypoxic environments may upregulate FGF23 transcription, which is represented by an increase in cFGF23, along with a commensurate increase in FGF23 cleavage resulting in normal bioactive iFGF23 levels [35]. Observational human studies also support a hypoxia-FGF23 relationship. A single center study of 32 pediatric patients demonstrated that preoperative oxygen saturation level negatively correlated with cFGF23 levels, suggesting that hypoxemia may also regulate FGF23 transcription in humans [36]. We now demonstrate that individuals with severe sleep disordered breathing are more likely to have elevated cFGF23 levels, but not iFGF23 levels.

Severe sleep disordered breathing is characterized by multiple overnight respiratory events associated with oxygen desaturation [37]. Prior reports of experiments conducted in cell cultures and animal models demonstrate that episodic hypoxia can directly impact the bone by increasing expression of HIF- $1 \alpha$ and stimulating FGF23 transcription $[38,39]$. Based on these data, we propose that intermittent nocturnal hypoxemia in sleep disordered breathing may directly trigger FGF23 transcription in the bone resulting in elevated cFGF23 levels. We further speculate that increases in FGF23 transcription induced by nocturnal hypoxemia in the absence of CKD is coupled to parallel increases in FGF23 cleavage, resulting in normal iFGF23 levels.

There are additional possible explanations for our findings. The elevation of cFGF23 in the severe sleep disordered breathing group may be a result of coexisting risk factors, such as elevated body mass index, inflammation, and hematologic indices in individuals with severe sleep disordered breathing. Prior investigations led by our group and others demonstrated significant associations between elevated body mass index and elevated FGF23 levels [40-45]. Indeed, adjustment for body mass index or waist circumference attenuated our results in both our multinomial logistic regression and linear regression analyses, and these covariates were the major confounders that rendered the relationship in the linear regression models non-significant. The mechanisms underlying the associations of elevated body mass index and FGF23 levels remain unknown, but an overlap between metabolic and inflammatory signals is known to exist, which may contribute to an inflammatory milieu in obesity [46]. Both acute and chronic inflammation are known stimuli of FGF23 transcription [47]. In all comparisons, including our subgroups of CKD and non-CKD, those with severe sleep disordered breathing had significantly higher CRP values than those without severe sleep disordered breathing. Although we adjusted for CRP in our models, inflammation remains a biologically plausible mechanism that may link sleep disordered breathing and elevated body mass index with FGF23 elevation.

Prior investigations also demonstrate that erythropoietin levels may be elevated in individuals with sleep disordered breathing [48] and data suggest that erythropoietin may directly regulate FGF23 production [16]. In addition, iron deficiency upregulates FGF23 transcription and may also be related to sleep related diseases [47, 49]. Although iron stores and erythropoietin are not available for our analyses, disturbances in hematologic parameters represent another mechanism by which cFGF23 levels may be elevated in severe sleep disordered breathing. Finally, prior investigations in humans demonstrated a relationship between obstructive sleep apnea and aberrant mineral metabolism [50-52]. By triggering alterations in the mineral metabolism axis, intermittent hypoxia may indirectly lead to FGF23 elevation through alterations in vitamin $\mathrm{D}$ and parathyroid hormone.

We acknowledge additional limitations in our study. Sleep disordered breathing exemplifies a good model of intermittent hypoxemia, but differs from models of chronic hypoxemia. Each model of hypoxemia associates with different biological responses [53], and the relationship of each of these to FGF23 homeostasis is unknown. We demonstrate small and likely not clinically meaningful elevations in cFGF23 levels in individuals with intermittent hypoxia. Whether chronic hypoxia models would demonstrate larger differences in cFGF23 levels is unknown. Human models of chronic hypoxia however, also have limitations. For example, clinical scenarios of severe lung disease are characterized as high inflammatory states, and a major etiology of lung disease includes smoking, another known risk factor associated with elevated FGF23 levels [54]. Due to the small sample size of the CKD subgroup, we could not elucidate the impact of severe sleep disordered breathing on FGF23 transcription or cleavage in CKD. Although we were able to adjust for important confounders such as body mass index and CRP in our cross-sectional analyses, we were unable to adjust for iron indices and do not have longitudinal FGF23 measurements available. Finally, our study only included Hispanic/ Latino populations. Given that prior studies have demonstrated racial variation in FGF23 levels [55], the generalizability of our findings may be limited.

In settings of increased FGF23 transcription but normal FGF23 cleavage, which result in isolated cFGF23 elevation, we do not yet understand what role c-terminal FGF23 fragments have given that the biologic activity and maladaptive cardiovascular effects of FGF23 are believed to be mediated by elevation in iFGF23 levels. However, elevated levels of cFGF23 and iFGF23 both demonstrate associations with morbidity and mortality in the general population and in those with CKD $[7,9,10]$. It is critical to continue investigations that help elucidate factors that regulate FGF23 production and cleavage. The current findings demonstrate that, similar to iron deficiency and inflammation, severe sleep disordered breathing may be another clinical state characterized by cFGF23 elevation in the absence of iFGF23 elevation. Further human studies in alternative models of hypoxemia and in larger CKD populations are needed to confirm our findings and ascertain the impact of CKD on cFGF23 and iFGF23 levels in sleep disordered breathing.

\section{Conflict of interest statement}

All authors have read the journal's policy on disclosure of potential conflicts of interest. RM has interest in Abbot Laboratories, AbbVie, Inc. and Teva Pharmaceuticals Industries Ltd. TI has received honoraria from Bayer and grant support from Shire. MW has received research support, honoraria or consultant fees from Amgen, Ardelyx, DiaSorin, Keryx, Lilly, Pfizer, Shire and Ultragenyx.

The authors thank the staff and participants of HCHS/SOL for their important contributions.

Investigators website - http://www.cscc.unc.edu/hchs/. This study was supported by grants R01DK081374 (MW), K24DK093723 (MW), R01DK102438 (TI), National Kidney Foundation of Illinois Young Investigator Grant (RM), and a Strategically Focused Research Network Center Grant from the American Heart Association (MW). The Hispanic Community Health Study/Study of Latinos was carried out as a collaborative study supported by contracts from the National Heart, Lung, and Blood Institute (NHLBI) to the University of North Carolina (N01HC65233), University of Miami (N01-HC65234), Albert Einstein College of Medicine (N01-HC65235), Northwestern University (N01HC65236), and San Diego State University (N01-HC65237). The following Institutes/Centers/Offices contribute to the HCHS/SOL through a transfer of funds to the NHLBI: National Institute on Minority Health and Health Disparities, National Institute on Deafness and Other Communication Disorders, National Institute of Dental and Craniofacial Research, National Institute of Diabetes and Digestive and Kidney Diseases, National Institute of Neurological Disorders and Stroke, NIH Institution-Office of Dietary Supplements. 


\section{Acknowledgements}

Each author contributed important intellectual content during manuscript drafting or revision and accepts accountability for the overall work by ensuring that questions pertaining to the accuracy or integrity of any portion of the work are appropriately investigated and resolved. The corresponding author confirms that he or she has had full access to the data in the study and final responsibility for the decision to submit for publication.

\section{Appendix A. Supplementary data}

Supplementary data to this article can be found online at https:// doi.org/10.1016/j.bone.2018.06.024.

\section{References}

[1] M. Wolf, Update on fibroblast growth factor 23 in chronic kidney disease, Kidney Int. 82 (7) (Oct 2012) 737-747.

[2] T. Isakova, P. Wahl, G.S. Vargas, et al., Fibroblast growth factor 23 is elevated before parathyroid hormone and phosphate in chronic kidney disease, Kidney Int. 79 (12) (Jun 2011) 1370-1378.

[3] C. Faul, A.P. Amaral, B. Oskouei, et al., FGF23 induces left ventricular hypertrophy, J. Clin. Invest. 121 (11) (Nov 2011) 4393-4408,

[4] A. Grabner, A.P. Amaral, K. Schramm, et al., Activation of cardiac fibroblast growth factor receptor 4 causes left ventricular hypertrophy, Cell Metab. 22 (6) (Dec 1 2015) 1020-1032.

[5] A. Alonso, F.L. Lopez, K. Matsushita, et al., Chronic kidney disease is associated with the incidence of atrial fibrillation: the Atherosclerosis Risk in Communities (ARIC) study, Circulation 123 (25) (Jun 28 2011) 2946-2953.

[6] O.M. Gutierrez, J.L. Januzzi, T. Isakova, et al., Fibroblast growth factor 23 and left ventricular hypertrophy in chronic kidney disease, Circulation 119 (19) (May 19 2009) 2545-2552.

[7] O.M. Gutierrez, M. Mannstadt, T. Isakova, et al., Fibroblast growth factor 23 and mortality among patients undergoing hemodialysis, N. Engl. J. Med. 359 (6) (Aug 7 2008) 584-592.

[8] J. Kendrick, A.K. Cheung, J.S. Kaufman, et al., FGF-23 associates with death, cardiovascular events, and initiation of chronic dialysis, J. Am. Soc. Nephrol. 22 (10) (Oct 2011) 1913-1922.

[9] J.J. Scialla, M. Wolf, Roles of phosphate and fibroblast growth factor 23 in cardiovascular disease, Nat. Rev. Nephrol. 10 (5) (May 2014) 268-278.

[10] J.J. Scialla, H. Xie, M. Rahman, et al., Fibroblast growth factor-23 and cardiovascular events in CKD, J. Am. Soc. Nephrol. 25 (2) (Feb 2014) 349-360.

[11] R. Mehta, X. Cai, J. Lee, et al., Association of fibroblast growth factor 23 with atrial fibrillation in chronic kidney disease, from the Chronic Renal Insufficiency Cohort Study, JAMA Cardiol. 1 (5) (Aug 01 2016) 548-556.

[12] P.L. Lutsey, A. Alonso, E. Selvin, et al., Fibroblast growth factor-23 and incident coronary heart disease, heart failure, and cardiovascular mortality: the Atherosclerosis Risk in Communities study, J. Am. Heart Assoc. 3 (3) (Jun 10 2014) e000936.

[13] E.G. Farrow, X. Yu, L.J. Summers, et al., Iron deficiency drives an autosomal dominant hypophosphatemic rickets (ADHR) phenotype in fibroblast growth factor23 (Fgf23) knock-in mice, Proc. Natl. Acad. Sci. U. S. A. 108 (46) (Nov 15 2011) E1146-E1155.

[14] M. Wolf, T.A. Koch, D.B. Bregman, Effects of iron deficiency anemia and its treatment on fibroblast growth factor 23 and phosphate homeostasis in women, J. Bone Miner. Res. 28 (8) (Aug 2013) 1793-1803.

[15] E.R. Smith, M.M. Cai, L.P. McMahon, S.G. Holt, Biological variability of plasma intact and C-terminal FGF23 measurements, J. Clin. Endocrinol. Metab. 97 (9) (Sep 2012) 3357-3365.

[16] E.L. Clinkenbeard, E.G. Farrow, L.J. Summers, et al., Neonatal iron deficiency causes abnormal phosphate metabolism by elevating FGF23 in normal and ADHR mice, J. Bone Miner. Res. 29 (2) (Feb 2014) 361-369.

[17] H. Fox, H.C. Purucker, I. Holzhacker, et al., Prevalence of sleep-disordered breathing and patient characteristics in a coronary artery disease cohort undergoing cardiovascular rehabilitation, J. Cardiopulm. Rehabil. Prev. 36 (6) (Nov/Dec 2016) $421-429$.

[18] M. Turhan, A. Bostanci, S. Bozkurt, Estimation of cardiovascular disease from polysomnographic parameters in sleep-disordered breathing, Eur. Arch. Otorhinolaryngol. 273 (12) (Dec 2016) 4585-4593.

[19] Z. Ehsan, S.L. Ishman, T.R. Kimball, et al, Longitudinal cardiovascular outcomes of sleep disordered breathing in children: a meta-analysis and systematic review, Sleep 40 (3) (Mar 01 2017).

[20] S. Stadler, T. Zimmermann, F. Franke, et al., Association of sleep-disordered breathing with diabetes-associated kidney disease, Ann Med. (Mar 27 2017) 1-9.

[21] C.L. Phillips, I. Laher, B.J. Yee, Is the kidney yet another potential end-organ casualty of obstructive sleep apnea? Am. J. Respir. Crit. Care Med. 192 (7) (Oct 01 2015) $779-781$.

[22] S.M. Dharia, M.L. Unruh, L.K. Brown, Central sleep apnea in kidney disease, Semin. Nephrol. 35 (4) (Jul 2015) 335-346.
[23] Y.C. Lee, S.Y. Hung, H.K. Wang, et al., Sleep apnea and the risk of chronic kidney disease: a nationwide population-based cohort study, Sleep 38 (2) (Feb 01 2015) 213-221.

[24] J. Xu, I.Y. Yoon, H.J. Chin, The effect of sleep apnea on all-cause mortality in nondialyzed chronic kidney disease patients, Sleep Med. 27-28 (Nov-Dec 2016) 32-38.

[25] Y.S. Lin, P.H. Liu, S.W. Lin, et al., Simple obstructive sleep apnea patients without hypertension or diabetes accelerate kidney dysfunction: a population follow-up cohort study from Taiwan, Sleep Breath. 21 (1) (Mar 2017) 85-91.

[26] T. Shimada, I. Urakawa, T. Isakova, et al., Circulating fibroblast growth factor 23 in patients with end-stage renal disease treated by peritoneal dialysis is intact and biologically active, J. Clin. Endocrinol. Metab. 95 (2) (Feb 2010) 578-585.

[27] L.M. Lavange, W.D. Kalsbeek, P.D. Sorlie, et al., Sample design and cohort selection in the Hispanic Community Health Study/Study of Latinos, Ann. Epidemiol. 20 (8) (Aug 2010) 642-649.

[28] P.D. Sorlie, L.M. Aviles-Santa, S. Wassertheil-Smoller, et al., Design and implementation of the Hispanic Community Health Study/Study of Latinos, Ann. Epidemiol. 20 (8) (Aug 2010) 629-641.

[29] S. Redline, D. Sotres-Alvarez, J. Loredo, et al., Sleep-disordered breathing in Hispanic/Latino individuals of diverse backgrounds. The Hispanic Community Health Study/Study of Latinos, Am. J. Respir. Crit. Care Med. 189 (3) (Feb 01 2014) $335-344$.

[30] V.L. Ernster, Nested case-control studies, Prev. Med. 23 (5) (Sep 1994) 587-590.

[31] H.M. Gonzalez, W. Tarraf, C.J. Rodriguez, et al., Cardiovascular health among diverse Hispanics/Latinos: Hispanic Community Health Study/Study of Latinos (HCHS/SOL) results, Am. Heart J. 176 (Jun 2016) 134-144.

[32] W.W. Wong, G. Strizich, M. Heo, et al., Relationship between body fat and BMI in a US hispanic population-based cohort study: results from HCHS/SOL, Obesity (Silver Spring) 24 (7) (Jul 2016) 1561-1571.

[33] L.A. Inker, C.H. Schmid, H. Tighiouart, et al., Estimating glomerular filtration rate from serum creatinine and cystatin C, N. Engl. J. Med. 367 (1) (Jul 5 2012) 20-29.

[34] T. Isakova, H. Xie, W. Yang, et al., Fibroblast growth factor 23 and risks of mortality and end-stage renal disease in patients with chronic kidney disease, JAMA 305 (23) (Jun 15 2011) 2432-2439.

[35] M. Wolf, K.E. White, Coupling fibroblast growth factor 23 production and cleavage: iron deficiency, rickets, and kidney disease, Curr. Opin. Nephrol. Hypertens. 23 (4) (Jul 2014) 411-419.

[36] M.R. Hanudel, K. Wesseling-Perry, B. Gales, et al., Effects of acute kidney injury and chronic hypoxemia on fibroblast growth factor 23 levels in pediatric cardiac surgery patients, Pediatr. Nephrol. 31 (4) (Apr 2016) 661-669.

[37] Y. Leng, C.T. McEvoy, I.E. Allen, K. Yaffe, Association of sleep-disordered breathing with cognitive function and risk of cognitive impairment: a systematic review and meta-analysis, JAMA Neurol. 74 (10) (Oct 1 2017) 1237-1245.

[38] C.M. Swanson, S.A. Shea, K.L. Stone, et al., Obstructive sleep apnea and metabolic bone disease: insights into the relationship between bone and sleep, J. Bone Miner. Res. 30 (2) (Feb 2015) 199-211.

[39] Q. Zhang, M. Doucet, R.E. Tomlinson, et al., The hypoxia-inducible factor-1alpha activates ectopic production of fibroblast growth factor 23 in tumor-induced osteomalacia, Bone Res. 4 (2016) 16011.

[40] M.A. Mirza, J. Alsio, A. Hammarstedt, et al., Circulating fibroblast growth factor-23 is associated with fat mass and dyslipidemia in two independent cohorts of elderly individuals, Arterioscler. Thromb. Vasc. Biol. 31 (1) (Jan 2011) 219-227.

[41] S. Zaheer, I.H. de Boer, M. Allison, et al., Fibroblast growth factor 23, mineral metabolism, and adiposity in normal kidney function, J. Clin. Endocrinol. Metab. 102 (4) (Apr 1 2017) 1387-1395.

[42] X. Hu, X. Ma, Y. Luo, et al., Associations of serum fibroblast growth factor 23 levels with obesity and visceral fat accumulation, Clin. Nutr. 37 (1) (Feb 2018) 223-228.

[43] L.J. Hanks, K. Casazza, S.E. Judd, N.S. Jenny, O.M. Gutierrez, Associations of fibroblast growth factor-23 with markers of inflammation, insulin resistance and obesity in adults, PLoS One 10 (3) (2015) e0122885.

[44] H. Ge, J. Zhang, Y. Gong, et al., Fibroblast growth factor receptor 4 (FGFR4) deficiency improves insulin resistance and glucose metabolism under diet-induced obesity conditions, J. Biol. Chem. 289 (44) (Oct 31 2014) 30470-30480.

[45] F.N. Ali, B. Falkner, S.S. Gidding, et al., Fibroblast growth factor-23 in obese, normotensive adolescents is associated with adverse cardiac structure, J. Pediatr. 165 (4) (Oct 2014) 738-743 (e731).

[46] C.N. Lumeng, A.R. Saltiel, Inflammatory links between obesity and metabolic disease, J. Clin. Invest. 121 (6) (Jun 2011) 2111-2117.

[47] V. David, A. Martin, T. Isakova, et al., Inflammation and functional iron deficiency regulate fibroblast growth factor 23 production, Kidney Int. 89 (1) (Jan 2016) $135-146$.

[48] X.B. Zhang, Y.M. Zeng, H.Q. Zeng, H.P. Zhang, H.L. Wang, Erythropoietin levels in patients with sleep apnea: a meta-analysis, Eur. Arch. Otorhinolaryngol. 274 (6) (Jun 2017) 2505-2512.

[49] M. Zilberman, D.S. Silverberg, I. Bits, et al., Improvement of anemia with erythropoietin and intravenous iron reduces sleep-related breathing disorders and improves daytime sleepiness in anemic patients with congestive heart failure, Am. Heart J. 154 (5) (Nov 2007) 870-876.

[50] C. Liguori, A. Romigi, F. Izzi, et al., Continuous positive airway pressure treatmen increases serum vitamin D levels in male patients with obstructive sleep apnea, J. Clin. Sleep Med. 11 (6) (Jun 15 2015) 603-607.

[51] A. Barcelo, C. Esquinas, J. Pierola, et al., Vitamin D status and parathyroid hormone levels in patients with obstructive sleep apnea, Respiration 86 (4) (2013) 295-301.

[52] N.C. Bozkurt, E. Cakal, M. Sahin, et al., The relation of serum 25-hydroxyvitamin-D levels with severity of obstructive sleep apnea and glucose metabolism abnormalities, Endocrine 41 (3) (Jun 2012) 518-525. 
[53] N.R. Prabhakar, G.L. Semenza, Adaptive and maladaptive cardiorespiratory responses to continuous and intermittent hypoxia mediated by hypoxia-inducible factors 1 and 2, Physiol. Rev. 92 (3) (Jul 2012) 967-1003.

[54] M.G. Vervloet, A.D. van Zuilen, A.C. Heijboer, et al., Fibroblast growth factor 23 is associated with proteinuria and smoking in chronic kidney disease: an analysis of the MASTERPLAN cohort, BMC Nephrol. 13 (Apr 24 2012) 20.

[55] A. Jovanovich, M. Chonchol, A.K. Cheung, et al., Racial differences in markers of mineral metabolism in advanced chronic kidney disease, Clin. J. Am. Soc. Nephrol. 7 (4) (Apr 2012) 640-647. 